\title{
Clinical and Radiographic Evaluation of the Operative Treatment of Lateral Humeral Condylar Fracture in Children at Shafa Hospital,
} Tehran, Iran

\author{
Davod Jafari, ${ }^{1}$ Samad Joudi, ${ }^{1,}$ and Payam Hassany Shariat Panahy ${ }^{2}$ \\ ${ }^{1}$ Bone and Joint Reconstruction Research Center, Shafa Orthopedic Hospital, Iran University of Medical Sciences, Tehran, Iran \\ ${ }^{2}$ Universiti Putra Malaysia, Serdang, Selangor, 43400, Malaysia \\ "Corresponding author: Samad Joudi, Bone and Joint Reconstruction Research Center, Shafa Orthopedic Hospital, Iran University of Medical Sciences, Tehran, Iran. Tel: \\ +98-9127314450, E-mail: samadjoudi@yahoo.com.sg
}

Received 2016 June 03; Revised 2016 November 13; Accepted 2016 November 14.

\begin{abstract}
Background: The goal of treatment in displaced lateral condylar fracture is to achieve union without residual deformity. However, growth disturbance may occur despite initial anatomic reduction and secure fixation.

Objectives: The purpose of this study was to evaluate clinical and radiographic results and assess complications associated with the operative treatment of lateral condylar fractures in children.

Methods: A total of 73 pediatric patients under the age of 13 years, who were diagnosed with displaced lateral condylar fractures of the humerus and were treated with open reduction and internal fixation, were assessed from March 2011 to September 2015 . The mean follow-up duration in these patients was 22 months ( 8 - 48 months). The clinical results were evaluated using the Hardcare scoring system. Also, anteroposterior and lateral radiographic views were assessed to identify the union process and presence of complications.

Results: The results of evaluation on 48 boys and 25 girls demonstrated that lateral condylar fractures of the humerus were more common in boys, aged 5-8 years (average: 57.1\%). There was no significant relationship between complications and sex. However, according to the radiographic evaluation, while fractures in 71 (97.2\%) patients were completely united without any complications, $2(2.8 \%)$ patients experienced overgrowth of the lateral humeral condyle. Clinical evaluation demonstrated excellent and good outcomes in 68 and 5 patients, respectively. Also, evaluation of Hardcare scores revealed no significant relationship between complications and clinical outcomes.

Conclusions: The present results were in line with previous research regarding the absence of complications such as non-union, avascular necrosis, fishtail deformity, cubitus varus, cubitus valgus, and pain. The findings demonstrated that the acceptable treatment option for displaced and rotated fractures is open reduction and internal fixation. Furthermore, deformity did not affect the clinical outcomes of fractures.
\end{abstract}

Keywords: Lateral Condylar Fractures of the Humerus (LCH), Union, Delayed Union, Overgrowth, Avascular Necrosis, Children

\section{Background}

Lateral humeral condyle fractures are common problems, affecting the elbow joint in children (1). These fractures account for nearly $5 \%-20 \%$ of pediatric elbow fractures and are ranked second place between supracondylar and medial epicondylar fractures (2-4) Lateral condyle mass fractures occur most commonly at $5-10$ years of age (3). The fracture line starts laterally in the metaphysis and extends between the condyles towards the elbow joint through the largely cartilaginous epiphysis. These fracture lines may or may not actually extend into the joint and pull the extensor muscles to cause displacement (5).

Lateral condylar physeal fractures can be classified by either the anatomic location of the fracture line or the extent of displacement. The most common classification for lateral humeral condyle fractures is based on the degree of displacement between fragments (6). Weiss et al. (6) has classified fractures in accordance with the fracture segment displacement and disruption of the cartilaginous hinge (Figure 1). Type I fractures are displaced less than 2 $\mathrm{mm}$, type II fractures are displaced more than $2 \mathrm{~mm}$ but have an intact cartilaginous hinge, and type III fractures are displaced more than $2 \mathrm{~mm}$ and do not have an intact cartilaginous hinge. Furthermore, 60\% of all fractures involving the lateral condylar physis are classified as type II and III fractures.

While the pediatric elbow is most often incompletely ossified when the fracture occurs, diagnosis of injury and detection of its severity require appropriate treatment; indeed, proper treatment leads to fewer fracture complications. Consequently, proper diagnosis and adequate treat- 


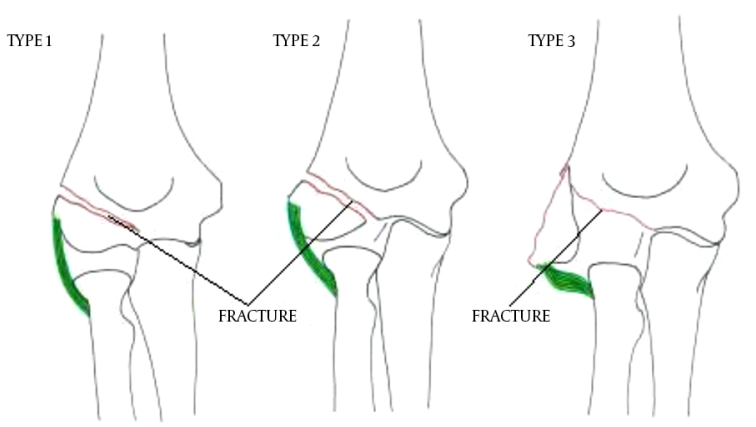

CLASSIFICATION OF LATERAL CONDYLE FRACTURE

Figure 1. Lateral Condyle Classification (Weiss, 2009)

ment might prevent serious complications in the elbow joint and preserve the neurological function of the arm and hand (7). Lateral condylar fractures may be managed with immobilization alone with cast, closed reduction and percutaneous pinning, or open reduction and internal fixation, with respect to the extent of displacement and degree of instability (8).

The treatment goal in lateral condyle fractures is achieving union without residual complications. According to a previous study, concurrent use of open reduction and internal fixation is the optimal procedure for displaced and rotated lateral condylar fractures of the humerus in children (1). However, despite initial anatomic reduction and secure fixation, complications such as nonunion, avascular necrosis, premature epiphyseal fusion, lateral condylar overgrowth, elbow stiffness, cubitus varus or cubitus valgus, and fishtail deformity may occur after the operative treatment of lateral condylar fractures (9).

According to the literature review, the outcomes of open reduction and internal fixation for lateral humeral condyle fractures have not been recently investigated in Iran. Therefore, in this study, we retrospectively studied the clinical and radiological outcomes of open reduction and internal fixation as the initial treatment option for displaced fractures in pediatric patients. As recommended by Lemme et al. (7), we believe that review of these injuries at a single medical center would help identify and assess treatment approaches and their outcomes.

\section{Objectives}

The purpose of this study was to evaluate the clinical and radiographic results, including complications associated with lateral condylar fracture treatment in children below 13 years of age, who underwent operative treatment at Shafa hospital, Tehran, Iran.

\section{Methods}

This retrospective study was carried out on all patients, admitted to the orthopedic department of Shafa hospital, Tehran, Iran between March 2011 and September 2015 in order to evaluate the clinical and radiographic results of lateral condylar fracture of humerus. We only included children who were below 13 years of age and had displaced fractures of the lateral humeral condyle. The subjects were treated with open reduction and internal fixation within three days following the trauma; the minimum of postoperative follow-up was six months.

The study was approved by the Institutional Review Board of Iran University of Medical Sciences. After obtaining the parents' informed consents, children underwent clinical examination, and radiographic views of the involved elbow were obtained.

The demographic and clinical data were entered in a database constructed for this study, and the information was analyzed, using SPSS Version 23 (IBM Corporation, Armonk, NY). Statistical analysis was performed, using Chisquare test and Fisher's exact test. Hardcare scoring system was applied for the evaluation of the clinical results (1) (Table 1), and anteroposterior and lateral radiographic views were obtained to evaluate the radiographic results.

\section{Results}

In this study, patients with type II and III lateral condylar fractures of the humerus underwent operative treatment. A total of 73 patients, including 48 (66\%) boys and 25 (34\%) girls within the age range of 1 - 12 years, were evaluated (average age: 6.9 years for boys and 7 years for girls); the observed difference was statistically significant ( $\mathrm{P}=$ 0.04 ). The patients were divided into three age groups of 1-4, 5-8, and 9-12 years in which 12 (16\%), 41 (56\%), and 20 (28\%) fractures were reported, respectively; the findings indicated a higher rate of fracture occurrence in the age group of 5-8 years (Table 2 ).

The mean follow-up duration was 22 months (8 - 48 months) and union was observed in all 73 patients in the final follow-up. No cases of avascular necrosis, fishtail deformity, non-union, cubitus valgus, cubitus varus, or spur were encountered. Complete union without any complications was reported in 71 (97.2\%) patients. Nevertheless, among 73 patients, obvious lateral condylar overgrowth of the distal humerus was observed in the radiographic evaluation of $2(2.8 \%)$ boys in the age groups of $1-4$ and 5 - 8 years, respectively; however, no functional abnormality was evident. There was no significant relationship between treatment-related complications, sex, and age group $(\mathrm{P}=0.3)$ (Table 3 ). 
Table 1. Hardcare Scoring System for the Clinical Evaluation (Kwang et al., 2010)

\begin{tabular}{|c|c|c|c|}
\hline Score & Range of Motion & Carrying Angle & Symptom \\
\hline \multirow{2}{*}{ Excellent } & \multirow{2}{*}{ Full range of motion } & \multirow{2}{*}{ Normal carrying angle } & No symptom \\
\hline & & & Complete healed fracture \\
\hline \multirow{2}{*}{ Good } & Efficient range of motion & \multirow{2}{*}{ Mild and subtle deformity } & No arthritis or neurological symptom \\
\hline & Loss of extension less than 15 degrees & & Completely healed fracture \\
\hline \multirow{2}{*}{ Poor } & \multirow{2}{*}{$\begin{array}{l}\text { Loss of motion to the extent of } \\
\text { disability }\end{array}$} & Alteration of the carrying angle & Arthritis or neurological symptom \\
\hline & & Permanent deformity & Non-union or avascular necrosis \\
\hline
\end{tabular}

Table 2. Characteristics of the Patients ${ }^{\mathrm{a}}$

\begin{tabular}{|c|c|c|c|c|}
\hline \multirow[t]{2}{*}{ Sex } & \multirow[t]{2}{*}{ No. (\%) } & \multicolumn{3}{|c|}{ Age Group, y } \\
\hline & & 1- 4 & $5-8$ & 9-12 \\
\hline Boys & $48(66)$ & $10(20)$ & $22(46)$ & $16(34)$ \\
\hline Girls & $25(34)$ & $2(8)$ & $19(76)$ & $4(16)$ \\
\hline Total & $73(100)$ & $12(16)$ & $41(56)$ & $20(28)$ \\
\hline
\end{tabular}

${ }^{\mathrm{a}}$ Values are expressed as No. (\%).

Table 3. The Relationship Between Complications, Sex, and Age Group

\begin{tabular}{|c|c|c|c|c|c|c|}
\hline \multirow[t]{2}{*}{ Union Process } & \multirow[t]{2}{*}{ Patients, No. (\%) } & \multirow[t]{2}{*}{ Boys, No. } & \multirow[t]{2}{*}{ Girls, No. } & \multicolumn{3}{|c|}{ Age Group, y } \\
\hline & & & & $1-4$ & $5-8$ & 9-12 \\
\hline Union without complication & $71(97.2)$ & 46 & 25 & 11 & 40 & 20 \\
\hline Overgrowth & $2(2.8)$ & 2 & 0 & 1 & 1 & 0 \\
\hline
\end{tabular}

Table 4 summarizes the relationship between Hardcare score and several factors such as gender, age group, and complications. The clinical results using the scoring system by Hardcare et al. were excellent in 68 (93\%) patients and good in 5 (7\%) patients; also, no poor results were reported in the subjects. Furthermore, 16.2\%, 55.9\%, and $27.9 \%$ of cases with excellent scores were in the age groups of $1-4,5-8$, and $9-12$ years, respectively.

All the girls (100\%) showed excellent clinical results, while $89.6 \%$ of the boys demonstrated excellent clinical results. Based on the findings, Hardcare score was not significantly associated with the age group or $\operatorname{sex}(\mathrm{P}=0.9)$. In cases with excellent clinical results, two patients had lateral condylar overgrowth, and all cases with good results experienced no complications. Statistical evaluation revealed no significant relationship between Hardcare score and overgrowth of lateral humeral condyle $(\mathrm{P}=0.5)$.

\section{Discussion}

Lateral condylar fractures of the humerus are one of the most common fractures in children. Open anatomical reduction and internal fixation with K-wires, followed by cast immobilization are the gold standard treatment for displaced fractures in children, as this treatment strategy leads to excellent clinical and radiological results (10). Although this treatment is very effective with excellent functional results, few complications may affect the clinical outcomes.

In terms of gender, the present results revealed that $66 \%$ of the patients were male. This finding was consistent with previous research, indicating male predominance in lateral condylar fractures in comparison with females $(3,4$, 8 ). Considering age, fractures mostly occurred in the age group of 5 - 8 years, which is in congruence with some previous studies $(3,4,8)$. However, we could not find any similar studies to make further comparisons; therefore, it is essential to determine which type of fracture is more common in boys and this age range. 
Table 4. The relationship between Hardcare score and sex, age, and complications ${ }^{\mathrm{a}}$

\begin{tabular}{|c|c|c|c|c|c|c|c|}
\hline \multirow[t]{2}{*}{ Hardcare Score } & \multirow[t]{2}{*}{ Union Without Complication } & \multirow[t]{2}{*}{ Overgrowth } & \multirow[t]{2}{*}{ Boys } & \multirow[t]{2}{*}{ Girls } & \multicolumn{3}{|c|}{ Age Group, y } \\
\hline & & & & & $1-4$ & $5-8$ & $9-12$ \\
\hline Excellent & $68(93)$ & 2 & 43 & 25 & 16.2 & 55.9 & 28.9 \\
\hline Good & $5(7)$ & 0 & 5 & 0 & 20 & 60 & 20 \\
\hline Poor & 0 & 0 & 0 & 0 & 0 & 0 & 0 \\
\hline
\end{tabular}

${ }^{\mathrm{a}}$ Values are expressed as No. (\%).

The main finding of the present study was the relationship between treatment with open reduction and internal fixation and complications. While in the current study, radiographic overgrowth of lateral condyle was noted in $2.8 \%$ of the patients, Marcheix et al. (11) reported an incidence rate of $7 \%$ in patients. Furthermore, according to Koh et al. (9), lateral overgrowth is the most common residual deformity following lateral condylar fractures.

A study by Pirbaz (12) reported the presence of lateral spur in nearly $73 \%$ of pediatric patients, whereas in our follow-up, no spur was observed on radiographs. The present study confirmed the results of previous research, which revealed fewer complications such as non-union, avascular necrosis, fishtail deformity, cubitus valgus, cubitus varus, and persistent pain in patients undergoing surgical treatment (2).

In this study, clinical outcomes based on the Hardcare scoring system were excellent in $93 \%$ of the patients and good in $7 \%$ of the cases in the final follow-up. While Marcheix et al. (11) showed excellent, good, and poor results in $67 \%, 13 \%$, and $20 \%$ of the patients, comparison of the findings indicated a higher rate of excellent scores in our patients. Also, two cases of heterotopic ossification were reported without any clinical or functional consequences. Unfortunately, we could not find any similar studies in this area for further comparisons.

One of the limitations of this study was that fractures were treated by different surgeons, despite the use of consistent treatment protocols. Also, the retrospective design of the study, short-term follow-up of distal humeral fractures, radiographic study of the carrying angle, and possible measurement bias are the other shortcomings of this research.

Taken together, it can be concluded that excellent clinical results and radiographic union can be achieved in children with lateral condylar fractures via operative treatment. Lateral overgrowth was found to be the most common complication. The results of this study can be applied as a significant and useful guideline to help orthopaedic departments offer high-quality operations while considering the associated complications. Also, these findings can be used as a reference for future prospective studies with similar research intentions.

\section{Acknowledgments}

The authors would like to thank all the patients who took part in this study. We also express our gratitude to the medical personnel of the orthopedic surgery ward of Shafa Orthopedic hospital for cooperating with us and providing facilities to perform this study.

\section{Footnote}

Authors' Contribution: Study concept and design: Davod Jafari and Samad Joudi; analysis and interpretation of data: Payam Hassany Shariat Panahy; drafting of the manuscript: Samad Joudi and Payam Hassany Shariat Panahy; and critical revision of the manuscript for important intellectual content and study supervision: Davod Jafari.

\section{References}

1. Song KS, Shin YW, Oh CW, Bae KC, Cho CH. Closed reduction and internal fixation of completely displaced and rotated lateral condyle fractures of the humerus in children. J Orthop Trauma. 2010;24(7):434-8. doi: 10.1097/BOT.ob013e3181de014f. [PubMed: 20577074].

2. Sharma H, Chirputkar K, Duncan RDD. Management of lateral humeral condylar mass fractures in children. Curr Orthop. 2007;21(2):145-9. doi: 10.1016/j.cuor.2007.03.004.

3. Sharma H, Sibinski M, Sherlock DA. Outcome of lateral humeral condylar mass fractures in children associated with elbow dislocation or olecranon fracture. Int Orthop. 2009;33(2):509-14. doi: 10.1007/s00264-007-0463-1. [PubMed: 17940766].

4. Leonidou A, Chettiar K, Graham S, Akhbari P, Antonis K, Tsiridis E, et al. Open reduction internal fixation of lateral humeral condyle fractures in children. A series of 105 fractures from a single institution. Strategies Trauma Limb Reconstr. 2014;9(2):73-8. doi: 10.1007/s11751-0140193-z. [PubMed: 25022896].

5. Jakob R, Fowles JV, Rang M, Kassab MT. Observations concerning fractures of the lateral humeral condyle in children. J Bone Joint Surg Br. 1975;57(4):430-6. [PubMed: 1104630]. 
6. Weiss JM, Graves S, Yang S, Mendelsohn E, Kay RM, Skaggs DL. A new classification system predictive of complications in surgically treated pediatric humeral lateral condyle fractures. $J$ Pediatr Orthop. 2009;29(6):602-5. doi:10.1097/BPO.0b013e3181b2842c. [PubMed: 19700990].

7. Lemme K, Lubicky JP, Zeni A, Riley E. Pediatric lateral condyle humeral fractures with and without associated elbow dislocations: a retrospective study. Am J Orthop (Belle Mead NJ). 2009;38(9):453-6. [PubMed: 19911099].

8. Herzenberg. Fractures of the Lateral Humeral The Pediatric Orthopedic Soxiety of North Amrica; 2014. Available from: www.POSNA.org.

9. Koh KH, Seo SW, Kim KM, Shim JS. Clinical and radiographic results of lateral condylar fracture of distal humerus in children.J Pediatr Or- thop. 2010;30(5):425-9. doi: 10.1097/BPO.ob013e3181df1578. [PubMed: 20574257].

10. Omid R, Choi PD, Skaggs DL. Supracondylar humeral fractures in children.JBone Joint Surg Am. 2008;90(5):1121-32. doi: 10.2106/JBJS.G.01354. [PubMed: 18451407].

11. Marcheix PS, Vacquerie V, Longis B, Peyrou P, Fourcade L, Moulies D. Distal humerus lateral condyle fracture in children: When is the conservative treatment a valid option? Orthop Traumatol Surg Res. Elsevier Masson SAS. 2011;97(3):304-7. doi: 10.1016/j.otsr.2010.10.007.

12. Pribaz JR, Bernthal NM, Wong TC, Silva M. Lateral spurring (overgrowth) after pediatric lateral condyle fractures. J Pediatr Orthop. 2012;32(5):456-60. doi: 10.1097/BPO.0b013e318259ff63. [PubMed: 22706459]. 